\title{
NEW EXAMPLES OF INHOMOGENEOUS EINSTEIN MANIFOLDS OF POSITIVE SCALAR CURVATURE
}

\author{
Charles P. Boyer, Krzysztof Galicki, and Benjamin M. Mann
}

\begin{abstract}
A вstract. The purpose of this note is to announce the explicit construction of a new infinite family of compact inhomogeneous Einstein manifolds of positive scalar curvature in every dimension of the form $4 n-5$ for $n>2$. In fact, each manifold has two, non-homothetic, Einstein metrics of positive scalar curvature. Moreover, in every fixed dimension, these families each contain infinitely many distinct homotopy types. Each individual manifold has a Sasakian 3-structure and all of these examples are bi-quotients of unitary groups of the form $U(1) \backslash U(n) / U(n-2)$. In particular, when $n=3$, we obtain infinite subfamilies of mutually distinct homotopy types where each member of the subfamily is strongly inhomogeneous; that is, these Einstein manifolds are not even homotopy equivalent to any compact Riemannian homogeneous space.
\end{abstract}

\section{Introduction}

Differential geometers have long been interested in examples of compact Einstein manifolds. Classically, the first examples of such manifolds were certain Riemannian homogeneous spaces (see [Bes: pages 6-8] for this early history) and it has been more difficult to find inhomogeneous examples. The first examples of compact inhomogeneous Einstein manifolds with positive scalar curvature were found by Page $[\mathrm{Pa}]$ in 1979 and his construction was later generalized by Bérard Bergery [BB]. In 1985 Koiso and Sakane [KoiS1, KoiS2] gave examples of inhomogeneous Kähler-Einstein metrics of positive scalar curvature and of arbitrary cohomogeneity. Two years later Tian and Yau [TY] gave non-homogeneous Kähler-Einstein metrics on the del Pezzo surfaces $\mathbb{C P}^{2} \# k\left(-\mathbb{C P}^{2}\right)$ for $3 \leq k \leq 8$. Then, in 1990, Wang and Ziller showed that torus bundles over a product of Kähler-Einstein

1991 Mathematics Subject Classification. Primary 53C25.

Received October 11, 1993.

During the preparation of this work all three authors were supported by NSF grants. 
manifolds of positive scalar curvature admit Einstein metrics [WZ]. Combining the Wang-Ziller result with the results of Koiso-Sakane or Tian-Yau gives the first odd-dimensional examples of compact inhomogeneous Einstein metrics of positive scalar curvature. However, as the inhomogeneous Kähler-Einstein metrics in [KoiS1, KoiS2] and [TY] are obtained only implicitly, often just by establishing the existence result, the actual Einstein metrics for these first odd-dimensional examples are only known to exist implicitly.

The purpose of this note is to present a new explicit construction of infinite families of $(4 n-5)$-dimensional Einstein manifolds of positive scalar curvature which are not homogeneous.

Definition A. [BGM2] Let $n \geq 3$ and $\mathbf{p}=\left(p_{1}, \ldots, p_{n}\right) \in \mathbb{Z}_{+}^{n}$ be an $n$ tuple of non-decreasing, pairwise relatively prime, positive integers. Let $\mathcal{S}(\mathbf{p})$ be the left-right quotient of the unitary group $U(n)$ by $U(1) \times U(n-$ 2) $\subset U(n)^{2}=U(n)_{L} \times U(n)_{R}$ where the action is given by the formula

$$
\mathbb{W} \stackrel{(\tau, \mathbb{B})}{\longrightarrow}\left(\begin{array}{ccc}
\tau^{p_{1}} & & \\
& \ddots & \\
& & \tau^{p_{n}}
\end{array}\right) \mathbb{W}\left(\begin{array}{ll}
\mathbb{I}_{2} & \mathbb{O} \\
\mathbb{O} & \mathbb{B}
\end{array}\right)
$$

Here $\mathbb{W} \in U(n)$ and $(\tau, \mathbb{B}) \in U(1) \times U(n-2)$.

Equivalently, $\mathcal{S}(\mathbf{p})$ is the quotient of the complex Stiefel manifold $V_{n, 2}^{\mathbb{C}}$ of 2 -frames in $\mathbb{C}^{n}$ by the specific free left circle action which depends on $\mathbf{p}$.

Theorem B. [BGM2] Let $n \geq 3$ and $\mathbf{p}=\left(p_{1}, \ldots, p_{n}\right) \in \mathbb{Z}_{+}^{n}$ be an $n$-tuple of non-decreasing, pairwise relatively prime, positive integers. Then $\mathcal{S}(\mathbf{p})$ is a compact, simply connected, $(4 n-5)$-dimensional smooth manifold which admits an Einstein metric $\check{g}(\mathbf{p})$ of positive scalar curvature and a compatible Sasakian 3-structure. In addition, $\mathcal{S}(\mathbf{p})$ also admits a second Einstein metric of positive scalar curvature, $g_{1}(\mathbf{p})$, which is non-homothetic to $\check{g}(\mathbf{p})$. Furthermore, both $(\mathcal{S}(\mathbf{p}), \check{g}(\mathbf{p}))$ and $\left(\mathcal{S}(\mathbf{p}), g_{1}(\mathbf{p})\right)$ are inhomogeneous Einstein manifolds as long as $\mathbf{p} \neq(1, \ldots, 1)$.

Using Theorem B and techniques developed by Eschenburg [E] to study certain 7-dimensional bi-quotients of $S U(3)$ we compute the integral cohomology ring of $\mathcal{S}(\mathbf{p})$.

Theorem C. [BGM2] Let $n \geq 3$ and $\mathbf{p}=\left(p_{1}, \ldots, p_{n}\right) \in \mathbb{Z}_{+}^{n}$ be an $n$-tuple of non-decreasing, pairwise relatively prime, positive integers. Then, as 
rings,

$$
H^{*}(\mathcal{S}(\mathbf{p}), \mathbb{Z}) \cong\left(\frac{\mathbb{Z}\left[b_{2}\right]}{\left[b_{2}^{n}=0\right]} \otimes E\left[f_{2 n-1}\right]\right) / \mathcal{R}(\mathbf{p}) .
$$

Here the subscripts on $b_{2}$ and $f_{2 n-1}$ denote the cohomological dimension of each generator. Furthermore,

1. The relations $\mathcal{R}(\mathbf{p})$ are generated by $\sigma_{n-1}(\mathbf{p}) b_{2}^{n-1}=0$ and $f_{2 n-1} b_{2}^{n-1}=$ 0 .

2. $\sigma_{n-1}(\mathbf{p})=\sum_{j=1}^{n} p_{1} \cdots \hat{p_{j}} \cdots p_{n}$ is the $(n-1)^{\text {st }}$ elementary symmetric polynomial in the entries of $\mathbf{p}$.

Notice that Theorem $\mathrm{C}$ shows that $H^{2 n-2}(\mathcal{S}(\mathbf{p}) ; \mathbb{Z})=\mathbb{Z}_{\sigma_{n-1}(\mathbf{p})}$ and hence has the following corollary.

Corollary D. [BGM2] Both $(\mathcal{S}(\mathbf{p}), \check{g}(\mathbf{p}))$ and $\left(\mathcal{S}(\mathbf{p}), g_{1}(\mathbf{p})\right)$ give infinitely many non-homotopy equivalent simply-connected compact inhomogeneous Einstein manifolds of positive scalar curvature in dimension $4 n-5$ for every $n \geq 3$.

The inhomogeneous $\check{g}(\mathbf{p})$ and $g_{1}(\mathbf{p})$ metrics are given in [BGM2]. The 7-dimensional case illustrates just how far some of these examples are from homogeneous Einstein geometry. The following theorem follows directly from a theorem of Eschenburg $[\mathrm{E}]$ and Theorem C.

Theorem E. If $\sigma_{2}(\mathbf{p})=p_{1} p_{2}+p_{2} p_{3}+p_{3} p_{1} \equiv 2(\bmod 3)$ then $\mathcal{S}\left(p_{1}, p_{2}, p_{3}\right)$ is strongly inhomogeneous; that is, it is not homotopy equivalent to any compact Riemannian homogeneous space.

Notice that Theorem E is not vacuous. For example, we have the following corollary.

Corollary F. [BGM2] $\mathcal{S}(c, c+1, c+2)$ is strongly inhomogeneous for every positive odd integer $c$. Similarly, $\mathcal{S}(d, d+3, d+5)$ is strongly inhomogeneous for every positive odd integer $d \equiv 2(\bmod 6)$ such that $d$ is not divisible by 5 . Thus, there exist countable families of homotopically distinct 7 -dimensional strongly inhomogeneous compact simply connected Einstein manifolds of positive scalar curvature.

To our knowledge, the families in Corollary F (and many other similar $\mathcal{S}\left(p_{1}, p_{2}, p_{3}\right)$ families $)$ are the only known examples of strongly inhomogeneous compact Einstein manifolds of positive scalar curvature. In dimension 7 some of our manifolds are diffeomorphic, although not isometric, to some of Eschenburg's bi-quotients. For example, this is true for the 
$\mathcal{S}(c, c+1, c+2)$ family. However, many of our $\mathcal{S}\left(p_{1}, p_{2}, p_{3}\right)$ spaces are not even homotopy equivalent to any Eschenburg spaces. Finally, as was known to Eschenburg [E], for large $c, \mathcal{S}(c, c+1, c+2)$ also admits a metric of positive sectional curvature.

\section{Outline of proofs}

Understanding the geometric structure of 3-Sasakian manifolds is critical to making our constructions. Sasakian 3-structures were introduced in 1970 by Kuo $[\mathrm{Ku}]$ and studied in the early seventies by several Japanese geometers including Ishihara, Kashiwada, Konishi, and Sasaki [I, IKon, Ka, Kon, Sas]. Of particular interest to us is the fact that Kashiwada [Ka] proved that every 3-Sasakian manifold is Einstein. Our investigation of this geometry in terms of its connection to other quaternionic geometries [BGM1, BGM2] then led directly to the results stated above.

A Sasakian manifold is a Riemannian manifold $(M, g)$ with a unit Killing vector field $\xi$ such that the $(1,1)$ tensor $\Phi=\nabla \xi$, defined as the covariant derivative of $\xi$, satisfies

$$
\left(\nabla_{X} \Phi\right)(Y)=\eta(Y) X-g(X, Y) \xi
$$

Here $\eta$ denotes the 1 -form dual to $\xi$ with respect to $g$ and $X$ and $Y$ are any two vector fields on $M$. A manifold $(M, g)$ that has three such structures $\left\{\Phi^{a}, \xi^{a}, \eta^{a}\right\}_{a=1,2,3}$ such that

$$
g\left(\xi^{a}, \xi^{b}\right)=\delta^{a b} \text { and }\left[\xi^{a}, \xi^{b}\right]=2 \epsilon^{a b c} \xi^{c}
$$

is called a 3 -Sasakian manifold. The $\mathcal{S}(\mathbf{p})$ spaces defined above arise from a 3-Sasakian version of the reduction procedure analogous to the symplectic reduction of Marsden and Weinstein [MW], hyperkähler reduction of Hitchin et al. [HKLR], and quaternionic reduction of Galicki and Lawson [GL]. Namely, let $\left(M, g, \xi^{a}\right)$ be a 3-Sasakian manifold with a connected compact Lie group $G$ acting on $M$ by 3 -Sasakian isometries; that is, isometries that commute with the vector fields $\xi^{a}$ for $a=1,2,3$. One can define a unique 3-Sasakian momentum mapping

$$
\mu: M \longrightarrow \mathfrak{g}^{*} \otimes \mathbb{R}^{3},
$$

where $\mathfrak{g}^{*}$ is the dual of the Lie algebra of $G$, by setting

$$
<\mu^{a}, \tau>=\frac{1}{2} \eta^{a}\left(X^{\tau}\right)
$$

where $\tau \in \mathfrak{g}, X^{\tau}$ is the corresponding infinitesimal isometry, and $\langle\cdot, \cdot\rangle$ denotes the natural pairing on $\mathfrak{g} \times \mathfrak{g}^{*}$. Using standard techniques for Riemannian submersions we prove 
Theorem G. [BGM2] If $\hat{M}=\mu^{-1}(0) / G$ is a smooth manifold then it has a natural 3-Sasakian structure induced by the inclusion map $\iota: \mu^{-1}(0) \hookrightarrow M$ and the projection map $\pi: \mu^{-1}(0) \rightarrow \hat{M}$.

We use Theorem $\mathrm{G}$ to construct the $\mathcal{S}(\mathbf{p})$ examples. To begin take $M=$ $S^{4 n-1}$ to be the round unit sphere of dimension $4 n-1$. This is well-known to carry a Sasakian 3-structure coming from the canonical embedding $S^{4 n-1} \subset$ $\mathbb{H}^{n}$. The group of 3-Sasakian isometries is $S p(n) \subset S O(4 n)$ and we take $G=U(1)_{\mathbf{p}}$ to be in the maximal torus $T^{n}$ in $S p(n)$ contained in $U(n) \subset$ $S p(n)$, acting with weights $\mathbf{p}$. If $\mathbf{u}=\left(u_{1}, \ldots, u_{n}\right) \in \mathbb{H}^{n}$ is the quaternionic coordinate system on the sphere then $T^{n}$ can be represented as diagonal matrices and the momentum map can be written as

$$
\mu(\mathbf{u})=\sum_{\alpha=1}^{n} p_{\alpha} \bar{u}_{\alpha} i u_{\alpha}
$$

where $\bar{w}$ denotes the quaternionic conjugate of $w \in \mathbb{H}$ and " $i$ " is the quaternionic unit defining the inclusion $U(n) \subset S p(n)$. When the weights are chosen to be pairwise coprime then the quotient $\hat{M}=\mathcal{S}(\mathbf{p})$ is a smooth manifold with a natural Sasakian 3-structure given by Theorem G. For any such $\mathbf{p}$ we identify the level set of the momentum map

$$
N(\mathbf{p})=\mu^{-1}(0) \in S^{4 n-1}
$$

with the complex Stiefel manifold $V_{n, 2}^{\mathbb{C}}=\frac{U(n)}{U(n-2)}$, via a smooth map $F$, and the quotient manifold $\hat{M}=\mathcal{S}(\mathbf{p})$ with the bi-quotient of $U(n)$ given in Definition A. Thus, the metric $\check{g}(\mathbf{p})$ on $\mathcal{S}(\mathbf{p})$ is obtained explicitly and we have the following diagram

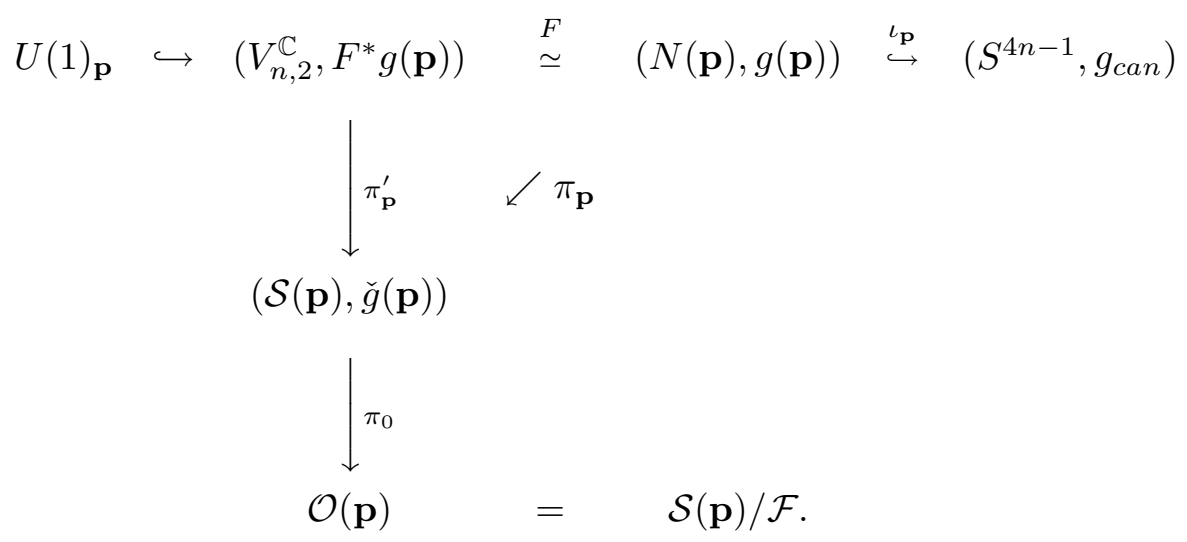


Here $\pi_{0}$ is the orbifold projection onto the quaternionic Kähler orbifold $\mathcal{O}(\mathbf{p})=\mathcal{S}(\mathbf{p}) / \mathcal{F}$, defined by the 3 -Sasakian foliation $\mathcal{F}$ which is given by the $S U(2)$ (or $S O(3)$ ) action generated by the vector fields $\xi^{a}$ of the 3 Sasakian structure [BGM2].

The results now follow from the general theory of 3-Sasakian manifolds presented in [BGM2].

\section{Acknowledgement}

We would like to thank Gerardo Hernandez, Jim Milgram, and Stephan Stolz for helpful discussions concerning this work. The second named author would also like to thank the Max-Planck Institute and its director Professor F. Hirzebruch for their hospitality and support.

\section{References}

[Bes] A. L. Besse, Einstein manifolds, Springer-Verlag, New York, 1987.

[BB] L. Bérard Bergery, Sur de nouvelles variétiés riemanniennes d'Einstein, Publications de l'Institut E. Cartan nô 4, Nancy, 1982, pp. 1-60.

[BGM1] C. P. Boyer, K. Galicki, and B. M. Mann, Quaternionic reduction and Einstein manifolds, Communications in Analysis and Geometry 1 (1993), no. 2, 1-51.

[BGM2] The Geometry and Topology of 3-Sasakian Manifolds, University of New Mexico and Max-Planck-Institute preprints, June 1993.

[E] J.H. Eschenburg, New examples of manifolds with strictly positive curvature, Invent. Math. 66 (1982), 469-480.

[GL] K. Galicki and B.H. Lawson, Jr., Quaternionic reduction and quaternionic orbifolds, Math. Ann. 282 (1988), 1-21.

[HKLR] N.J. Hitchin, A. Karlhede, U. Lindström, and M. Roček, Hyperkähler metrics and supersymmetry, Comm. Math. Phys. 108 (1987), 535-589.

[I] S. Ishihara, Quaternion Kählerian manifolds and fibered Riemannian spaces with Sasakian 3-structure, Kodai Math. Sem. Rep. 25 (1973), 321-329.

[IKon] S. Ishihara and M. Konishi, Fibered Riemannian spaces with Sasakian 3-structure, in honor of K. Yano, Kinokuniya, Tokyo, Differential Geometry (1972), 179194.

[Ka] T. Kashiwada, A note on a Riemannian space with Sasakian 3-structure, Nat. Sci. Reps. Ochanomizu Univ. 22 (1971), 1-2.

[KoiS1] N. Koiso and Y. Sakane, Nonhomogeneous Kähler-Einstein metrics on compact complex manifolds, Lecture Notes in Math., vol. 1201, Springer, 1986, pp. 165179.

[KoiS2] _ Nonhomogeneous Kähler-Einstein metrics on compact complex manifolds II, Osaka J. Math. 25 (1988), 933-959.

[Kon] M. Konishi, On manifolds with Sasakian 3-structure over quaternion Kählerian manifolds, Kodai Math. Sem. Reps. 26 (1975), 194-200.

[Ku] Y.-Y. Kuo, On almost contact 3-structure, Tôhoku Math. J. 22 (1970), 325332. 
[MW] J. Marsden and A. Weinstein, Reduction of symplectic manifolds with symmetry, Rep. Math. Phys. 5 (1974), 121-130.

[Pa] D. Page, A compact rotating gravitational instanton, Phys. Lett. 79B (1979), $235-238$.

[Sas] S. Sasaki, Spherical space forms with normal contact metric 3-structure, J. Diff. Geom. 6 (1972), 307-315.

[TY] G. Tian and S. T. Yau, Kähler-Einstein metrics on complex surfaces with $C_{1}>0$, Comm. Math. Phys. 112 (1987), 175-203.

[WZ] M. Wang and W. Ziller, Einstein metrics on principal torus bundles, J. Diff. Geom. 31 (1990), 215-248.

Department of Mathematics and Statistics, Univerity of New Mexico, A LBUQUERQUE, N M 87131

E-mail address: cboyer@math.unm.edu, galicki@math.unm.edu, and mann@math.unm.edu 